\title{
Performance Analysis of Alamouti Code MIMO-OFDM Systems for Error Control and IQ Impairments
}

\author{
Rajesh Bhadada, Ph.D \\ Department of ECE, \\ MBM Engineering College, \\ JNV University, Jodhpur (India)
}

\author{
Ritika Taparia \\ Department of ECE, \\ MBM Engineering College, \\ JNV University, Jodhpur (India)
}

\begin{abstract}
Bit Error Rate (BER) performance is the most important metric of any digital communication system. MIMO-OFDM systems designed with Alamouti Coding technique, using Error Control Coding schemes has been investigated under this study to achieve BER enhancement and scrutinized for Additive White Gaussian Noise (AWGN) Channel with Inphase and Quadrature-phase (IQ) impairments. This paper details out the model developed using NI LabVIEW and discusses simulation results comparing the error control coding RS, RS-CC and Convolution encoding with 4-PSK and 4-QAM.
\end{abstract}

\section{General Terms}

Bit Error Rate, Space Time Coding (STC).

\section{Keywords}

MIMO-OFDM, Alamouti Coding, IQ Gain Imbalance, Quadrature Skew

\section{INTRODUCTION}

MIMO-OFDM is a recent wireless technology and is considered in number of developing wireless standards. Information transmitted through a communication channel always encounters factors causing various impairments, thus errors get induced. Current digital transmission systems handle large amount of data and such impairments can result in undetected and uncorrected errors consequently degrading systems BER performance. To encounter the same, error control codes are employed in the system entailing detection and correction of errors. Assorted research challenges and design parameters of the MIMO-OFDM system have been addressed and explored by many. MATLAB simulation software has been widely used to evaluate its BER; we have used National Instrument's LabVIEW to analyze system's BER performance by incorporating features provided by the platform.

\subsection{Motivation}

In present times, demand for high data rate wireless access has been increasing very rapidly needing more bandwidth to be deployed to achieve the same. Many new techniques have emerged and one of the most promising wireless communication technologies is MIMO-OFDM which offers high data rate transmission capability along with robustness against various noise and channel induced impairments. These characteristics make it more attractive for many high data rate standard such as 4G, 5G, IEEE 802.16 (WAN), IEEE 802.11n (LAN) etc. [1]. A careful analysis of BER and its improvement is of vital importance for such techniques.

\subsection{MIMO-OFDM Overview}

MIMO-OFDM combines features and advantages of two prominent technologies i.e., Multiple Input Multiple Output (MIMO) and Orthogonal Frequency Division Multiplexing (OFDM). MIMO is a capacity enhancement scheme, based on multipath phenomena, i.e. transmission over wireless links via multiple antennas at transmitter and receiver. The main advantages are beam-forming, spatial diversity and spatial multiplexing [2]. MIMO systems may be implemented in a number of different ways; one of such effective procedure being space time coding (STC), where the multiple copies of information are being transmitted by encoding single bit through both space and time [3].

OFDM is principally a block modulation technique; it is a multi-carrier digital modulation method which converts a frequency selective channel into a parallel collection of frequency flat sub-channels. Under OFDM an input bit stream is split in $N$ parallel streams and each of them is modulated and transmitted via separate orthogonal subcarriers, thereby achieving better spectral efficiency [1] Therefore, MIMO-OFDM achieves strong link reliability in addition to being quite effective against multipath fading, inter symbol interference (ISI) and inter carrier interference (ICI).

\section{SYSTEM MODELLING}

To weave MIMO and OFDM, MIMO algorithms and antenna arrays are applied over OFDM technique to achieve capacity enhancement and diversity gain for broadband transmission [4]. In this study, error control codes were implemented in the MIMO-OFDM system and then BER was scrutinized under AWGN channel and IQ impairments. Figure 1 depicts a $2 \mathrm{X} 2$ MIMO-OFDM model which employs error control coding, and the key parts of aforesaid model are detailed next.

\subsection{Error Control Coding and Decoding}

Referring to Figure 1, incoming bits were initially coded using any error control coding or forward error correction coding technique. Thereafter at the receiving end a decoder was used to recover information. Under this study, three error control coding schemes i.e., Reed Solomon (RS) Codes, Convolution Codes and Concatenated RS-CC Codes were investigated one by one.

\subsection{Symbol Mapping and De-Mapping}

Post encoder, bit stream was mapped or modulated, converting bits into complex symbols. At the receiving end, vice-versa de-mapping or demodulation technique was used to recover bits. Under this work, Phase Shift Keying (PSK) and Quadrature Amplitude Modulation (QAM) based symbol mapping/de-mapping schemes were used. 


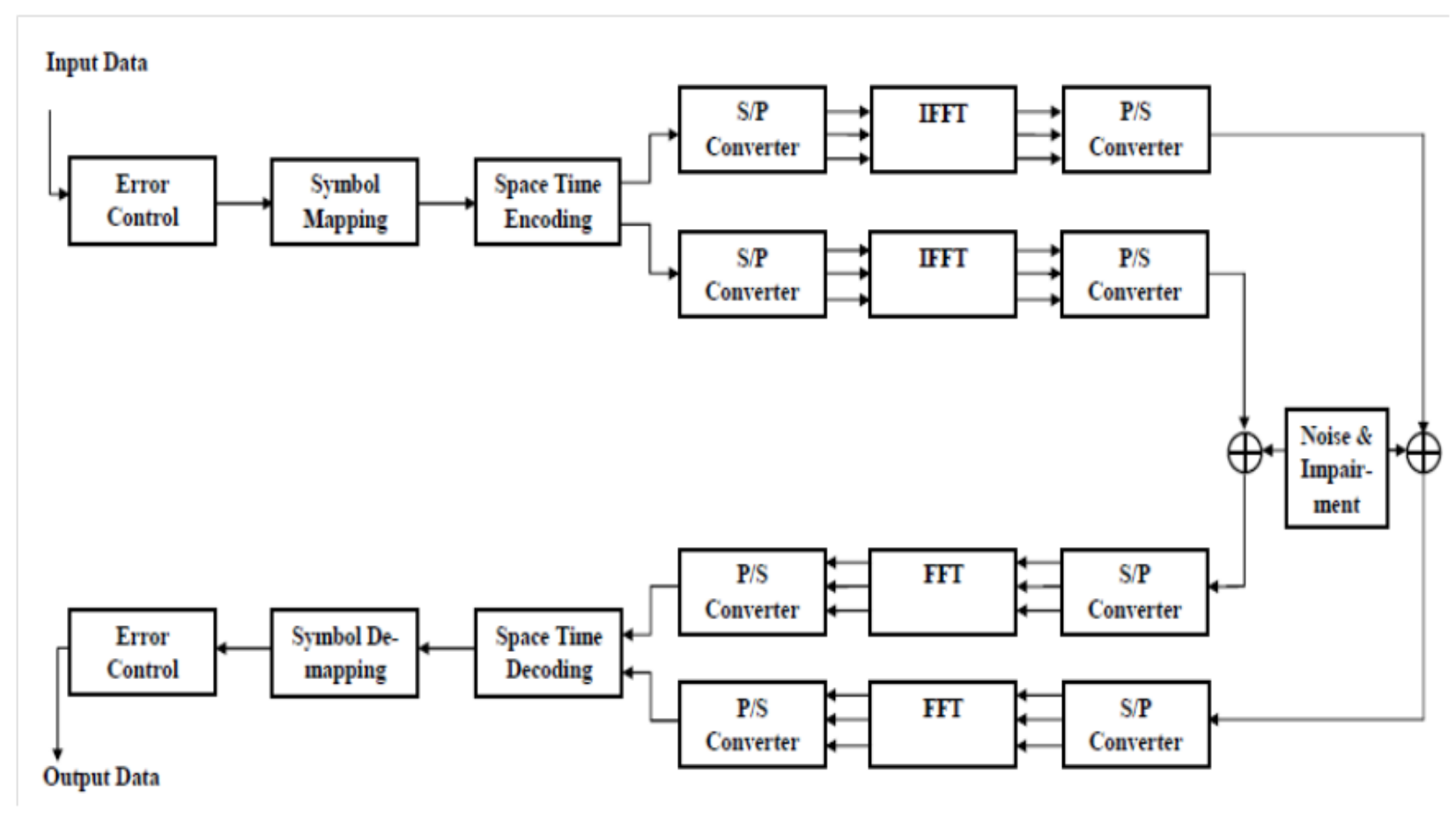

Fig 1: Block Diagram of 2X2 MIMO-OFDM System using Error Control Coding

\subsection{Space-Time Coding and Decoding}

Space-Time coding was designed for multiple antenna transmission and principally it adds redundancy in both spatial and temporal domains, i.e. blocks of same information were transmitted from each transmitting antenna in different order [6]. Space-Time Block Code called Alamouti Codes was employed here. They are also called Orthogonal Space Time Block Codes (OSTBC), which is a simple two branch transmit diversity scheme [7]. The encoding sequence $\mathrm{X}$ is given by:

$$
X=\left[\begin{array}{rr}
\mathrm{c} 1 & -\mathrm{c} 2^{\prime} \\
\mathrm{c} 2 & \mathrm{c} 1^{\prime}
\end{array}\right]
$$

where, $\mathrm{c1}^{\prime}$ and $\mathrm{c} 2$ ' are complex conjugate of symbol $\mathrm{c} 1$ and c2, which are symbols given as input to Alamouti encoder.

\subsection{OFDM Modulation and Demodulation}

To perform OFDM modulation, initially the incoming serial data was converted into parallel and pilot symbols were inserted. Thereafter Fast Fourier Transform (FFT) was applied at the transmitter for modulation and parallel stream of data was again converted into serial stream. At the receiver, such incoming serial data was converted into parallel and pilot symbols were removed and Inverse Fast Fourier Transform (IFFT) was used for OFDM demodulation, followed by conversion of the parallel streams of data into serial.

\subsection{Noise and Impairments}

AWGN channel and IQ impairments were simultaneously applied in this study to evaluate their effect on BER of system by varying test conditions; i.e. simple AWGN channel, AWGN with IQ Gain Imbalance Impairment and AWGN with Quadrature Skew Impairment.

\section{SIMULATION}

National Instruments LabVIEW was used to model and simulate a 2 X2 MIMO-OFDM system using aforementioned STC scheme. Due to print size limitations the complete scheme could not be included in this paper. However, a partial LabVIEW block diagram showing Alamouti Coding and decoding is shown in figure 2. Code rates for all error control coding schemes were kept consistent throughout the study; RS Codes had 4 bits per RS symbols, Convolution Codes with rate 1/3 and Concatenated RS-CC codes, RS Codes had rate of $11 / 15$.

Each test simulation was conducted with ten readings. Each test on a particular mapping technique was done with and without error control coding schemes. During simulation for each test performance 1000 bits were transmitted. The constellation size for both symbol mapping technique i.e. PSK and QAM was kept 4. BER with no error control coding scheme was compared with the BER obtained from system consecutively using aforesaid error control codes.

\section{RESULTS}

Comparison between bit error rates of different simulation results, obtained from various aforesaid test conditions, for MIMO-OFDM system based on Alamouti Coding Scheme are graphically presented and analyzed in this section. Figure $3 \mathrm{~A}$ and B illustrates BER comparative graphs depicting results obtained from simulation model when only AWGN Channel was used and $E_{b} / N_{o}$ was varied from $6 \mathrm{~dB}$ to $14 \mathrm{~dB}$. From figure $3 \mathrm{~A}$ it was observed that with convolution coding average BER reduction was $80.96 \%$ followed by RS-CC coding with $50.54 \%$ and RS coding with $29.63 \%$ and from figure $3 \mathrm{~B}$, average $\mathrm{BER}$ reduction was $78.15 \%$ for convolution coding, $45.99 \%$ for RS-CC coding and $31.04 \%$ for RS coding. 
Figure $4 \mathrm{~A}$ and $\mathrm{B}$ depicts BER comparative graphs illustrating results obtained from simulation model when AWGN
Channel with IQ Gain Imbalance Channel Impairment was

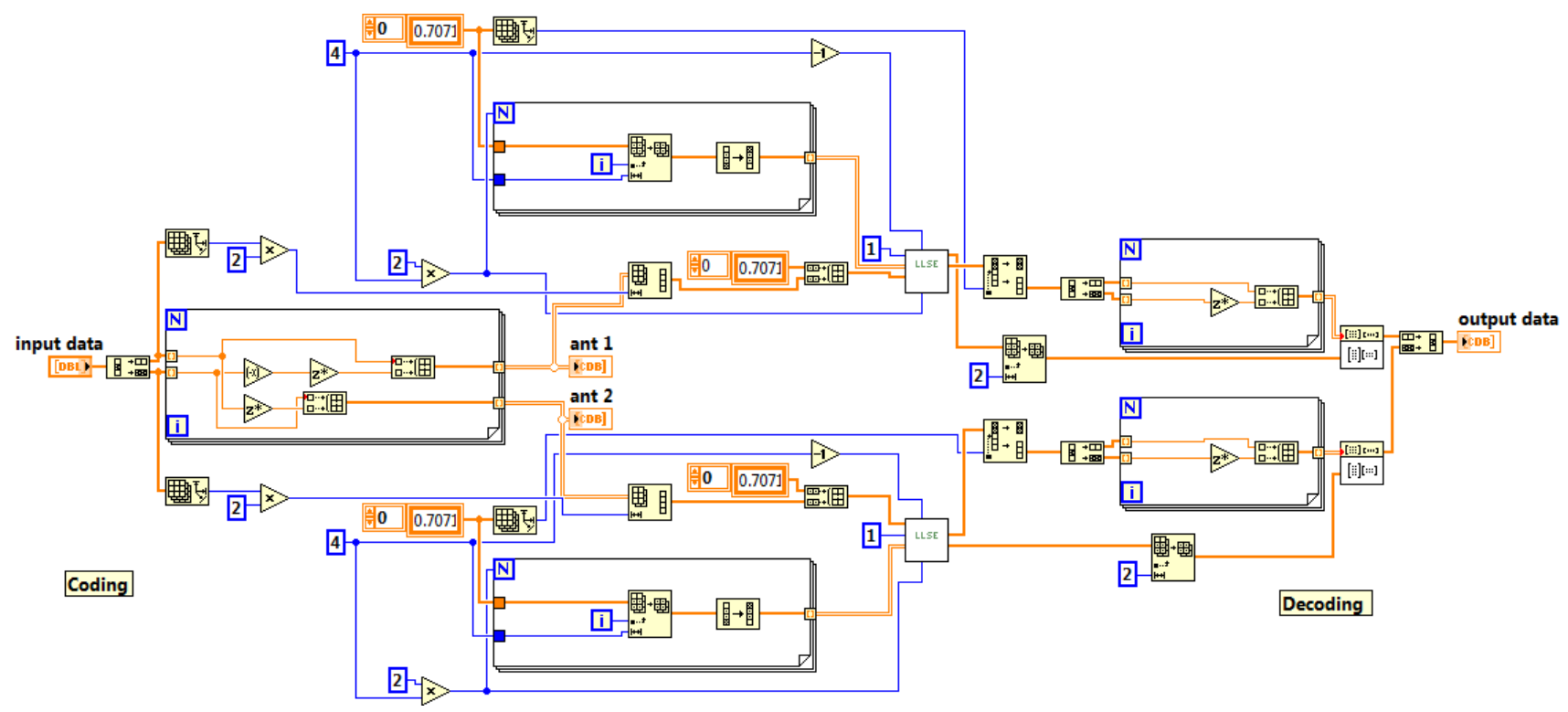

Fig 2: Partial LabVIEW Block Diagram of Alamouti Coding and Decoding.
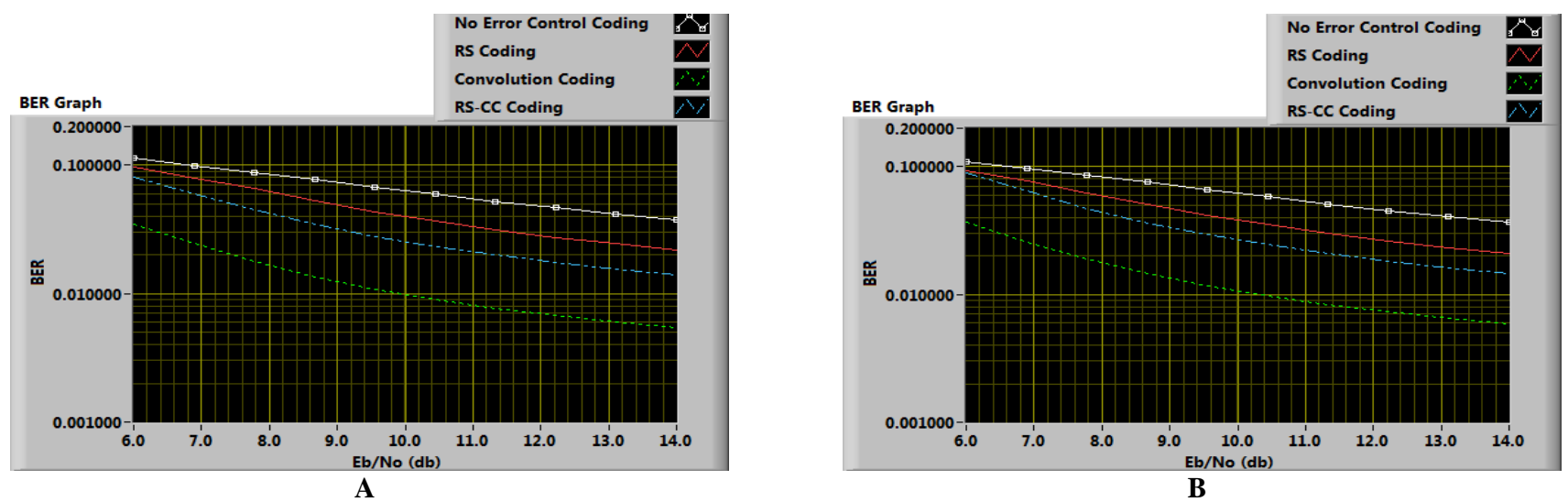

Fig. 3 A: BER comparisons for MIMO OFDM with 4-PSK and AWGN Channel.

B: BER comparisons for MIMO OFDM with 4-QAM and AWGN Channel.

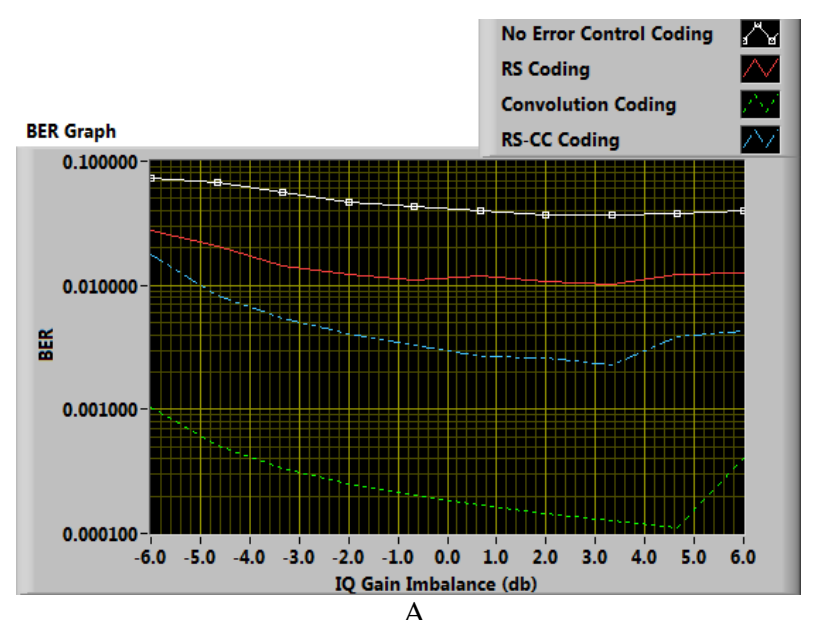

A

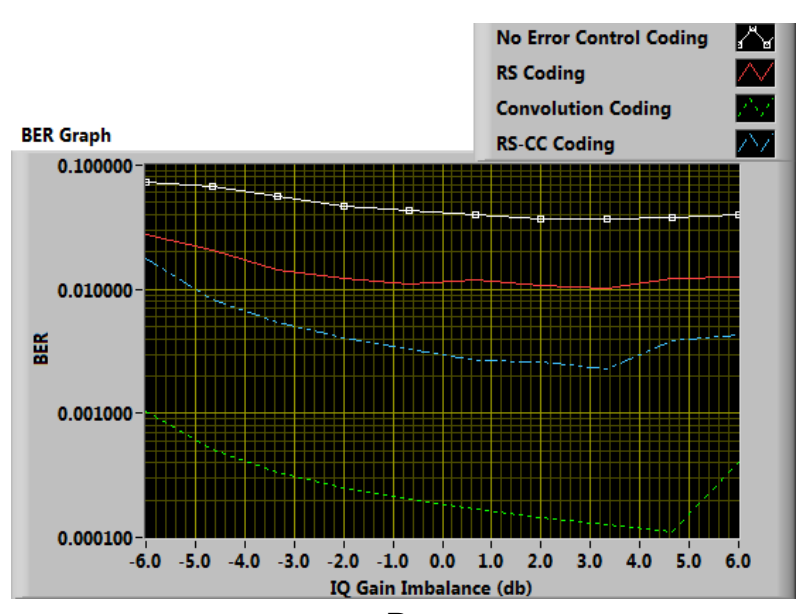

B

Fig. 4 A: BER comparisons for MIMO OFDM with 4-PSK and AWGN Channel with IQ Gain Imbalance. B: BER comparisons for MIMO OFDM with 4-QAM and AWGN Channel with IQ Gain Imbalance. 

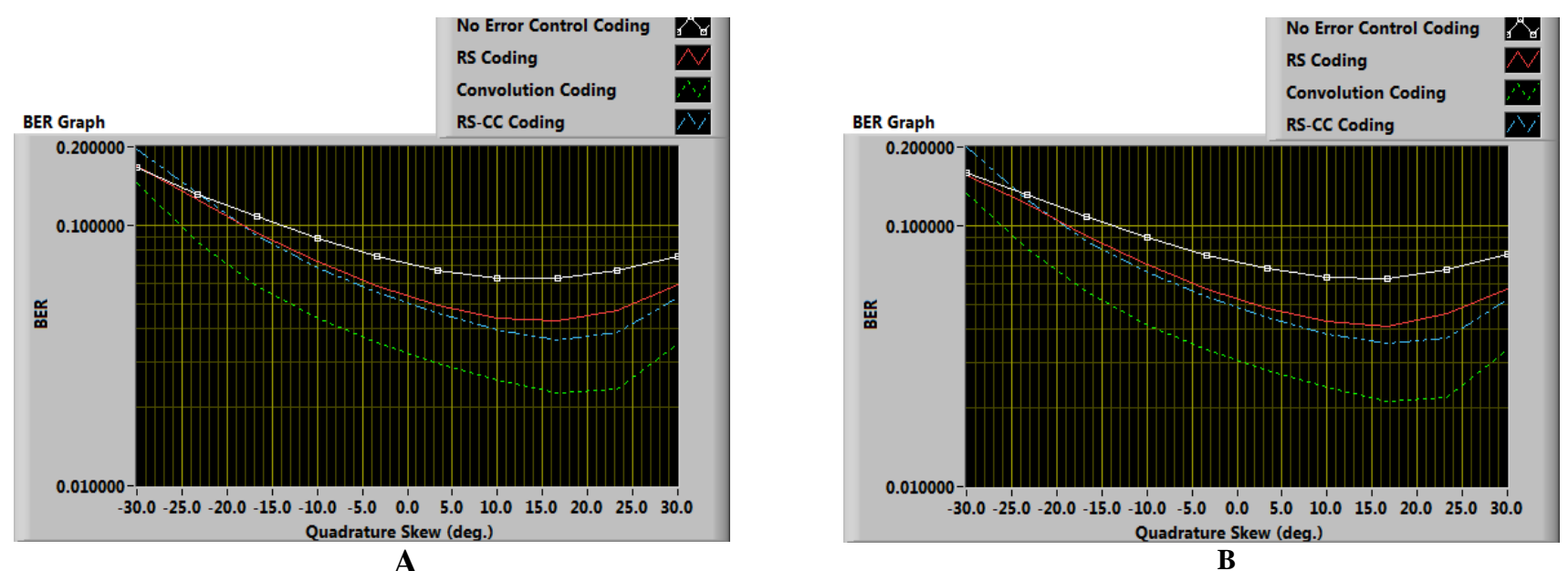

Fig. 5 A: BER comparisons for MIMO OFDM with 4-PSK and AWGN Channel with Quadrature Skew. B: BER comparisons for MIMO OFDM with 4-QAM and AWGN Channel with Quadrature Skew.

used and $\mathrm{E}_{\mathrm{b}} / \mathrm{N}_{\mathrm{o}}$ was kept fixed at $10 \mathrm{~dB}$ and IQ Gain Imbalance was varied from $-6 \mathrm{~dB}$ to $6 \mathrm{~dB}$. It was observed that average BER reduction, for figure 4A was $96.38 \%$ with convolution coding followed by RS-CC coding with $90.04 \%$ and RS coding with $61.34 \%$ and for figure 4B, $99.31 \%$ with convolution coding followed by RS-CC coding with $88.70 \%$ and RS coding with $70.01 \%$.

Figure $5 \mathrm{~A}$ and $\mathrm{B}$ demonstrates BER comparative graphs depicting results obtained from simulation model when AWGN Channel with Quadrature Skew Channel Impairment was used and $\mathrm{E}_{\mathrm{b}} / \mathrm{N}_{\mathrm{o}}$ was kept fixed at $10 \mathrm{~dB}$ and Quadrature Skew was varied from $-30^{\circ}$ to $30^{\circ}$. RS coding, convolution coding and RS-CC coding provided average BER reduction of $15.97 \%, 44.21 \%$ and $16.36 \%$ respectively as shown in figure $5 \mathrm{~A}$ and figure 5B shows that RS coding, convolution coding and RS-CC coding provided average BER reduction of $19.17 \%, 47.87 \%$ and $22.73 \%$ respectively.

\section{CONCLUSIONS}

Analysis of all the simulation results infers that by application of Error Control Coding in MIMO-OFDM systems, the BER performance can be improved as compared with un-coded systems. Figure 3, 4 and 5 depicting simulation results were observed and their comparative BER performance was scrutinized and it can be concluded that in all these scenarios, systems using Convolution coding were best performers followed by RS-CC coding and RS coding.

It was also observed that when 4-QAM modulation technique was employed, the average BER performance of the system was noted to be marginally better as compared to when 4-PSK modulation technique was used.

For further works, this model can be extended by increasing number of transmit or receive antenna, any other mapping and error control technique could be employed. For implementation of the system any other space time coding scheme, space frequency coding scheme and space time frequency coding scheme can be employed, as well as, behavior of system can also be observed for any other range of observation or any other channel impairment and noise.

\section{REFERENCES}

[1] Bolcskei Helmut, 2006 "MIMO-OFDM wireless systems: basics, perspectives, and challenges," Wireless Communications, IEEE 13, no. 4, pp. 31-37.

[2] Cortes-Pena, Luis Miguel, 2009"MIMO Space-Time Block Coding (STBC): Simulations and Results," Personal \& Mobile Communications, pp. 1-8.

[3] Gesbert David, Mansoor Shafi, Da-shan Shiu, Peter J. Smith, and Ayman Naguib, 2003 "From theory to practice: an overview of MIMO space-time coded wireless systems" Selected Areas in Communications, IEEE Journal no. 21, no. 3, pp. 281-302.

[4] Stuber Gordon L., John R. Barry, Steve W. Mclaughlin, Ye Li, Mary Ann Ingram, and Thomas G. Pratt, 2004 "Broadband MIMO-OFDM wireless communications," Proceedings of the IEEE 92, no. 2, pp. 271-294.

[5] Gupta Amit, Antonio Forenza, and Robert W. Heath, 2004 "Rapid MIMO-OFDM software defined radio system prototyping," In Signal Processing Systems, SIPS, IEEE Workshop, pp. 182-187.

[6] Alamouti Siavash, 1998 "A simple transmit diversity technique for wireless communications," Selected Areas in Communications, IEEE Journal no. 16, no. 8, pp. 1451-1458.

[7] Tarokh Vahid, Hamid Jafarkhani, and A. Robert Calderbank, 1999 "Space-time block codes from orthogonal designs," Information Theory, IEEE Transactions no. 45, no. 5, pp. 1456-1467.

[8] Lu Ben, Guosen Yue, and Xiaodong Wang, 2004 "Performance analysis and design optimization of LDPCcoded MIMO OFDM systems," Signal Processing, IEEE Transactions no. 52, no. 2, pp. 348-361.

[9] Zhang Wei, Xiang-Gen Xia, and Khaled Ben Letaief, 2007 "Space-time/frequency coding for MIMO-OFDM in next generation broadband wireless systems," Wireless Communications, IEEE 14, no. 3, pp. 32-43.

[10] Jiancai Liu, Shutin Chen, Jiakai Xu, 2013 "The LabVIEW Simulation of Space-Time Coding Technique in the MIMO-OFDM System", Remote Sensing Science, Volume 1, Issue 1, p.p.9-14. 